This journal is the official publication of Bangladesh Society of Physiologists (BSP)

Web URL: www.banglajol.info/index.php/JBSP

Abstracted /indexed in Index Copernicus, Director of Open Access Journal, HINARI Index Medicus for South East Asia Region, Google Scholar, 12OR, infobse index, Open J gate, Cite factor, Scientific indexing services

pISSN-1983-1213; e-ISSN-2219-7508

\title{
Article
}

Article information:
Received: 3rd Jan 2021
Accepted: 5 th Sept 2021
DOI: https://doi.org/10.3329/jbsp.v16i2.57565

Corresponding author:

Dr. Ayesha Akhter, Department of Physiology, Shaheed Monsur Ali Medical College, Dhaka, Email: shumiayesha@gmail.com

Cite this article:

Akhter A, Mostafa M, Sultana S, Ferdousi S. Inter relationship of sympathetic reflex response and oxidative stress in polycystic ovary syndrome. J Bngladesh Soc Physiol 2021;16(2): 61-69.

This article is open access licensed under CC BY NC SA which allows readers copy, distribute, display, and perform the work and make derivative works based on it only for noncommercial purposes.

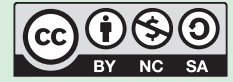

\section{Inter relationship of sympathetic reflex response and oxidative stress in polycystic ovary syndrome}

\author{
Ayesha Akhter ${ }^{1}$, Musfika Mostafa ${ }^{2}$, Shamima Sultana ${ }^{3}$, \\ Sultana Ferdousi ${ }^{3}$ \\ 1. Department of Physiology, Shaheed Monsur Ali Medical College, Dhaka \\ 2. Department of Physiology, Ibrahim Medical College, Dhaka \\ 3. Department of Physiology, Bangabandhu Sheikh Mujib Medical \\ University, Dhaka, Bangladesh.
}

\section{Abstract}

Background: Polycystic ovary syndrome (PCOS) is a very common reproductive endocrine disorder. Altered cardiovascular autonomic modulation and oxidative stress may predispose PCOS patients to cardiac events in the long term. Objective: To assess the relationship between sympathetic autonomic reactivity and oxidative stress in patients with polycystic ovary syndrome. Methods: This cross-sectional study was conducted in the Department of Physiology, Bangabandhu Sheikh Mujib Medical University (BSMMU), Shahbag, Dhaka from September 2018 to August 2019 on 35 newly diagnosed obese (BMI $\geq 25 \mathrm{~kg} / \mathrm{m}^{2}$ ) PCOS patients aged 20-35 years. Age and BMI matched 35 apparently healthy subjects were also enrolled. Two noninvasive conventional autonomic function tests, such as systolic blood pressure (SBP) fall during active standing and diastolic blood pressure (DBP) rise during sustained handgrip were conducted on all subjects for evaluation of sympathetic reactivity. For assessment of oxidative stress, plasma malondialdehyde level as oxidant and plasma catalase level as antioxidant were measured in all subjects by spectrophotometry. For statistical analysis Independent sample "t" test, Pearson's correlation test and multiple regression analysis were done. Results: In this study PCOS patients had significantly higher resting heart rate, SBP and DBP than healthy control $(\mathrm{p}<0.01)$. Fall of SBP during standing was significantly lower $(\mathrm{p}<0.05)$ while rise of DBP during 
sustained handgrip was significantly higher $(\mathrm{p}<0.05)$ in PCOS compared to healthy control. Plasma catalase level was significantly lower $(\mathrm{p}<0.01)$ and plasma malondialdehyde level significantly higher $(\mathrm{p}<0.001)$ in PCOS in comparison to healthy control. On correlation analysis, rise of DBP showed significant negative correlation $(\mathrm{p}<0.05)$ with plasma catalase level in PCOS. On multiple regression analysis rise of DBP showed significant negative $(\mathrm{p}<0.01)$ association with plasma catalase and significant positive $(\mathrm{p}<0.01)$ association plasma malondialdehyde in PCOS. Conclusion: Based on these results it is concluded that measures of sympathetic reactivity were related to oxidative stress in PCOS.

Keywords: Autonomic reactivity, polycystic ovary syndrome, oxidative stress, MDA, catalase

Introduction

$\mathbf{P}$ olycystic ovary syndrome (PCOS) is aendocrine disorder affecting reproductive function in women with a global prevalence of $8-13 \% .{ }^{1}$ It is the leading cause of anovulatory infertility in women. The implications of PCOS reaches beyond the reproductive sphere. Several metabolic co-morbidities such as obesity, insulin resistance, dyslipidaemia, hypertension and obstructive sleep apnea confer significant risk for poor cardiovascular health in these women in the long term. ${ }^{2-3}$ PCOS is currently recognizedby the presence of any two of itskey features- chronic absence of ovulation, clinical and/or biochemical signs of androgen excess and ovaries with multiple cysts on ultrasound. ${ }^{4}$

Disturbance in the balance between the activity of the sympathetic and parasympathetic system causing impaired autonomic balance predisposes them to various cardiovascular disorders. ${ }^{5-6}$ Heart rate variability (HRV) evaluated resting autonomic activity in PCOS and reported autonomic dysfunction. ${ }^{711} \mathrm{Few}$ authors assessed the status of autonomic reactivity in PCOS patients by conventional autonomic function tests. ${ }^{12-}$ ${ }^{13}$ Autonomic reactivity is described as the response of the ANS to various physiological provocations. ${ }^{6} \mathrm{Almost}$ all of these studies reported autonomic dysfunction characterized by parasympathetic insufficiency and sympathetic hyperactivity in PCOS compared to normal women. Very few authors found no alteration in cardiac autonomic tone analyzed by $\mathrm{HRV}$ in PCOS compared to healthy control. ${ }^{14-15}$

Conventional autonomic function tests (CAFT) were introduced by Ewing in 1982, for bedside evaluation of the integrity of autonomic reflex response to physiological provocations.For assessment of sympathetic reactivity, systolic blood pressure response (SBP) to active standing and diastolic blood pressure (DBP) response to sustained isometric handgrip (IHG) were recommended. Fall of SBP $\leq 10 \mathrm{~mm} \mathrm{Hg}$ within one minute of standing from supine position and rise of DBP $\geq 16 \mathrm{~mm}$ of $\mathrm{Hg}$ during IHG indicates intact sympathetic reactivity. ${ }^{16}$ Saranya and colleagues observed significantly increased rise of DBP during IHG in a group of PCOS patients when compared to control. ${ }^{12}$ But Zanella et al. found no such difference in rise of DBP between PCOS and control. ${ }^{13}$

Moreover, several studies have reported increased oxidative stress in PCOS patients which could be an additional risk factor for cardiovascular morbidity. ${ }^{17-20}$ Oxidative stress implies an imbalance between the production of

Volume 16 No. 2 December 2021: 61-69 
reactive oxygen species (ROS) and the antioxidant defense system of the body leading to damage of important biomolecules like DNA, lipids and proteins and ultimately cellular dysfunction and apoptosis. Peroxidation of polyunsaturated fatty acids by ROS yields a very stable and toxic compound known asmalondialdehyde (MDA). It is frequently measured as a biomarker of increased pro-oxidant levels in blood. ${ }^{21}$ High serum MDA level was observed in PCOS compared to healthy control. 19-20,22-23 Enzymatic antioxidants including superoxide dismutase, catalase and glutathione peroxidase are the first line of defense against oxidative stress in the body. Catalase is present in peroxisomes of all cells. It efficiently reduces $\mathrm{H}_{2} \mathrm{O}_{2}$ to water and $\mathrm{O}_{2}$, thus protecting the body from its harmful effects of $\mathrm{H}_{2} \mathrm{O}_{2} \cdot{ }^{21}$ Serum Catalase levels were lower in PCOS compared to age and BMI matched normal women in several studies. ${ }^{24-26}$

In certain chronic diseases, such as chronic kidney disease and essential hypertension, reduced HRV correlated with increased pro-oxidant levels. ${ }^{27-28}$ Though there is ample evidence that altered autonomic modulation and oxidative stress may contribute to cardiovascular morbidity in PCOS patients, the relationship between them is yet to be explored. Therefore this study aimed to investigate the possible relationship of oxidative stress and sympathetic autonomic reactivity in the pathogenesis of PCOS.

\section{Methods}

Study design, setting, participants

This cross-sectional study was carried out from September 2018 to August 2019 at the Department of Physiology, BSMMU, Shahbag, Dhaka to assess the relationship between cardiovascular autonomic reactivity and oxidative stress in 35 newly diagnosed PCOS patients, aged $20-35$ years with $\mathrm{BMI} \geq 25 \mathrm{~kg} / \mathrm{m}^{2}$. For comparison 35 age and BMI matched apparently healthy women with regular menstrual cycles were taken as control.

\section{Sampling}

The patients were enrolled from the outpatient department of Endocrinology, BSMMU by purposive sampling and the control subjects were recruited by personal contact. The protocol of this study was approved by the Institutional Review Board of BSMMU.

\section{Exclusion criteria}

All the subjects were free from any other systemic diseases. Women who were pregnant, lactating or on any hormonal therapy were not included in the study.

\section{Data collection procedure}

After briefing about the study, informed written consent was taken from each subject. Detailed personal and medical history was taken.

After enrollment, blood sample was collected from all the subjects with all aseptic precautions for estimation of plasma catalase and malondialdehyde (MDA) Plasma catalase and plasma MDA were measured by spectro-photometry. All subjects were advised to finish their meal by 9:00 pm on the night before and to avoid any type of stress or any sedative or hypnotic medications. They were also requested to take a light breakfast without tea and coffee the next morning and afterwards attend the autonomic nerve function laboratory in the Department of Physiology, BSMMU between 8:00 am to 10:00 am. A thorough physical examination was done and height, weight, hip and waist circumference of all subjects were measured. BMI and waist-hip ratio was calculated. Each of the subjects was briefed about the details of the procedures and encouraged to achieve maximum efficient performance. Two noninvasive cardiovascular reflex tests, BP response to sustained isometric hand grip and BP response to standing (orthostatic stress test) were conducted in the autonomic nerve function laboratory of the Department of Physiology, BSMMU, Dhaka.

The subject was advised to take rest for 15-20 minutes in a controlled laboratory environment after which her resting pulse and blood pressure were checked. The blood pressure was measured in supine position. Then she was asked to stand up suddenly and remain still for two minutes. 
Within one minute of standing her blood pressure was measured again and the fall of systolic blood pressure from the resting state was calculated. After this the subject was seated and her blood pressure was measured after 5 minutes of rest. Then she was given a dynamometer to grip by her dominant hand with maximum force. The dynamometer was then gripped at $30 \%$ of her maximum voluntary contraction (MVC) for as long as possible upto 3 minutes. Her blood pressure was measured again just before release of the grip from the non-dominant arm and rise of diastolic BP during the handgrip was calculated. All the BP measurements were done by a standard manual sphygmomanometer.

\section{Statistical analysis}

Data were expressed as Mean \pm SD. Statistical analysis was done by SPSS version 22 . Independent sample ' $t$ ' test was done for comparison of data between PCOS and control. Pearson's correlation coefficient test and multiple regression analysis were done to assess the relationship of conventional autonomic function variables with plasma catalase and MDA.A p value of $<0.05$ was considered as statistically significant.

\section{Results}

In this study all PCOS patients were comparable to healthy control by age, BMI and waist-hip ratio (Table I). Resting heart rate, SBP and DBP were significantly higher $(\mathrm{p}<0.01)$ in PCOS compared to control (Table I). PCOS patients had significantly lower fall of SBP $(\mathrm{p}<0.05)$ during standing and significantly higher $(\mathrm{p}<0.05)$ rise of DBP than healthy control (Figure1). In addition,

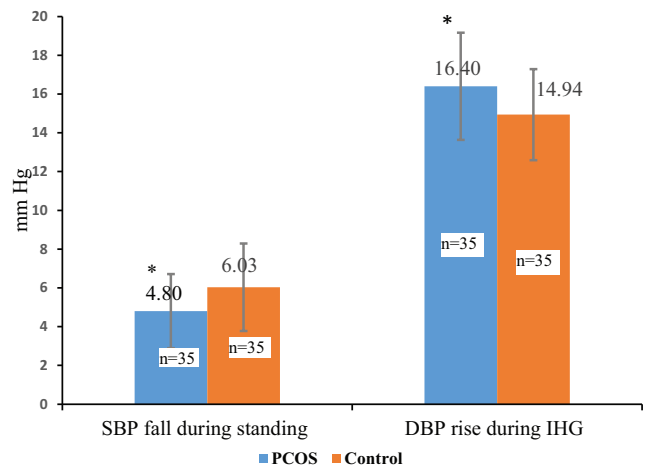

Figure 1: Mean \pm SD values of fall of SBP and rise of DBP in PCOS and healthy control $(\mathrm{N}=70)$. Statistical analysis was done by Independent sample t test; SBP-systolic blood pressure; DBP-diastolic blood pressure; N-total number of subjects; n-number of subjects in each group. $*=\mathrm{p}<0.05$.

Table I: Basal characteristics in PCOS and control $(\mathrm{N}=70)$

\begin{tabular}{lccc}
\hline Variable & $\operatorname{PCOS}(\mathrm{n}=35)$ & Control $(\mathrm{n}=35)$ & $\mathrm{p}$ value \\
\hline Age & $26.97 \pm 5.0$ & $27.69 \pm 4.00$ & 0.485 \\
(years) & $(20-35)$ & $(22-33)$ & 0.419 \\
BMI & $29.22 \pm 3.42$ & $28.63 \pm 2.56$ & \\
$\left(\mathrm{~kg} / \mathrm{m}^{2}\right)$ & $(25.09-38.28)$ & $(25.38-34.58)$ & 0.093 \\
Waist-Hip ratio & $0.88 \pm 0.06$ & $0.86 \pm 0.05$ & \\
& $(0.75-1.01)$ & $(0.76-0.96)$ & $0.001^{* *}$ \\
Resting heart rate & $88.14 \pm 8.88$ & $79.98 \pm 10.52$ & \\
(beats/min) & $(75-103.45)$ & $(60-96.77)$ & $0.001^{* *}$ \\
Resting SBP & $121.83 \pm 7.51$ & $(115.77 \pm 6.96$ & \\
(mmHg) & $(110-135)$ & $75.91 \pm 5.32$ & $0.001^{* *}$ \\
Resting DBP & $79.94 \pm 4.73$ & $(65-88)$ & \\
(mmHg) & $(70-88)$ & &
\end{tabular}

Data were expressed as Mean $\pm \mathrm{SD}$. Values in parentheses indicate ranges; Statistical analysis was done by Independent sample t-test; PCOS-polycystic ovary syndrome; BMI-Body Mass Index $\left(\mathrm{kg} / \mathrm{m}^{2}\right)$; SBP = Systemic blood pressure ; DBP = Diastolic blood pressure; N-total number of subjects; n-number of subjects in each group. 
plasma catalase levels were significantly lower and plasma MDA levels were significantly higher $(\mathrm{p}<0.01$ and $\mathrm{p}<0.001$ respectively) in PCOS when compared to healthy control (Table II).

Rise of DBP showed significant negative correlation $(\mathrm{p}<0.05)$ with plasma catalase in
PCOS (Table III). In multiple regression analysis, rise of DBP showed significant inverse relationship with plasma catalase levels $(\mathrm{p}<0.01)$ and significant positive relationship with plasma MDA $(\mathrm{p}<0.01)$ in PCOS patients (Table IV).

Table II: Plasma Catalase and MDA in PCOS and control groups (N=70)

\begin{tabular}{lccc}
\hline Variable & PCOS $(\mathrm{n}=35)$ & Control $(\mathrm{n}=35)$ & $\mathrm{p}$ value \\
\hline Plasma catalase & $172.06 \pm 64.88$ & $226.60 \pm 79.68$ & $0.002 * *$ \\
$(\mathrm{U} / \mathrm{ml})$ & $(59-282)$ & $(101-389)$ & \\
Plasma MDA & $541.88 \pm 223.35$ & $203.00 \pm 92.54$ & $0.000^{* * *}$ \\
$(\mathrm{ng} / \mathrm{ml})$ & $(101.30-952.50)$ & $(82.04-478.80)$ & \\
\hline
\end{tabular}

Data were expressed as Mean \pm SD. Values in parentheses indicate ranges; Statistical analysis was done by Independent sample t test; PCOS-polycystic ovary syndrome; MDA-Malondialdehyde; N-total number of subjects; n-number of subjects; $* *=p<0.01 ; * * *=p<0.001$.

Table III: Correlation of fall of SBP during standing and rise of DBP during isometric handgrip with plasma catalase levels in PCOS $(\mathrm{n}=35)$

\begin{tabular}{llcc}
\hline Variables & \multicolumn{2}{c}{ PCOS } \\
\cline { 2 - 4 } Dependent & Independent & r value & p value \\
\hline Fall of SBP $(\mathrm{mmHg})$ & Plasma Catalase & 0.107 & 0.542 \\
Rise of DBP $(\mathrm{mmHg})$ & & -0.346 & $0.041^{*}$ \\
\hline
\end{tabular}

Statistical analysis was done by Pearson correlation coefficient test. COS-polycystic ovary syndrome; SBPsystolic blood pressure; DBP-diastolic blood pressure; $n=$ number of subjects; $*=p<0.05$.

Table IV: Multiple regression analysis of rise of DBP (dependent variable) with plasma MDA and Catalase levels (independent variables) in $\operatorname{PCOS}(n=35)$

\begin{tabular}{lllllll}
\hline Rise of DBP & \multicolumn{2}{c}{ Coefficients } & & \multicolumn{2}{c}{$95 \%$ CI } & \multirow{2}{*}{ p value } \\
\cline { 2 - 3 } & $\mathrm{B}$ & $\beta$ & & Lower limit & Upper limit & \\
\hline Constant & 17.852 & - & & 15.415 & 20.288 & 0.000 \\
Plasma Catalase $(\mathrm{U} / \mathrm{ml})$ & -0.032 & -0.746 & & -0.049 & -0.015 & $0.001^{*}$ \\
Plasma MDA(ng/ml) & 0.007 & 0.599 & & 0.002 & 0.012 & $0.004^{*}$ \\
\hline
\end{tabular}

PCOS-polycystic ovary syndrome; MDA-malondialdehyde;CI-confidence interval; SBP-systolic blood pressure; DBP-diastolic blood pressure;n-number of subjects; ${ }^{* *}=\mathrm{p}<0.01$.

Volume 16 No. 2 December 2021: 61-69 


\section{Discussion}

The present study assessed sympathetic autonomic reactivity by two bedside conventional autonomic function tests and measured plasma catalase and MDA in newly diagnosed obese $\left(\mathrm{BMI} \leq 25 \mathrm{~kg} / \mathrm{m}^{2}\right)^{29}$ PCOS patients and compared the results with apparently healthy regularly menstruating age and BMI matched women.In this study, significantly higher resting heart rate, SBP and DBP were observed in PCOS patients compared to control. Saranya and colleagues reported a similar observation. They suggested that since resting heart rate and blood pressure are principally under vagal modulation and sympathetic control respectively, patients with PCOS might have decreased vagal tone and increased sympathetic tone, that is, an altered sympathovagal balance at rest. ${ }^{12}$ Hashim, Hamdan and Al-Salihi observed a higher resting heart rate but similar SBP and DBP in PCOS compared to control. ${ }^{7}$ But others found these measures comparable in PCOS and healthy control. ${ }^{10,11,19,22}$

Reflex response by sympathetic component was assessed by the orthostatic stress test and sustained handgrip test. The fall of SBP during standing and rise of DBP during isometric handgrip was normal in both groups. But the significant difference between the two groups regarding these two parameters suggests relatively higher sympathetic response in these patients. A similar significant rise of DBP during sustained IHG in PCOS patients compared to control was observed by Saranya and colleagues and they concluded that exaggerated sympathetic modulation was present in PCOS. ${ }^{12}$ On the contrary, in another study, rise of DBP during sustained handgrip, did not increase in obese and non-obese PCOS women when compared with age and BMI matched healthy women. ${ }^{13}$ Although, all the outcomes of sympathetic autonomic reactivity tests were within normal range in PCOS women as recommended by Ewing and Clarke ${ }^{16}$ comparison with healthy control revealed a significant alteration in autonomic response characterized by increased sympathetic reactivity.

In our study, significant inverse correlation between rise of DBP during handgrip exercise and plasma catalase levels in the present series of PCO Spatients suggests an increase in sympathetic reactivity was related to their low anti-oxidant status. In addition, significant negative association of rise of DBP was observed with plasma catalase after adjustment with plasma MDA levels in PCOS patients. Also significant positive relationship of rise of DBP was seen with plasma MDA levels after adjustment with plasma catalase levels in PCOS patients. These findings provide evidence that increased oxidant and decreased antioxidant level, consequently higher oxidative stress, is associated with enhanced sympathetic reactivity.

In the present study, the lower plasma catalase levels in PCOS patients agrees with other previous investigators suggesting a lower antioxidant status in these series of patients. ${ }^{17,24-}$ ${ }^{26}$ Kurdoglu and colleagues found no significant difference in catalase levels between hyperin sulinemic and normoinsulinemic non obese PCOS women. They also observed almost similar catalase levels in nonobese PCOS patients and BMI matched controls and concluded that antioxidant levels may not be decreased in nonobese PCOS women without insulin resistance. On the contrary, several authors found significantly higher plasma catalase levels in PCOS patients than control. This finding was explained by adequate counterbalance by a compensatory antioxidant response. ${ }^{30,31}$ In this study, the significantly higher levels of malondialdehyde (MDA) in PCOS patients agrees with the previous observations. ${ }^{17,19,20,22,23}$ But almost similar MDA levels in PCOS and healthy women was also reported. ${ }^{32-34}$

The precise influence of oxidative stress on autonomic nerve function is not clear but a body

Volume 16 No. 2 December 2021: 61-69 
of evidence in literature suggested that altered nitric oxide signaling in the central and peripheral nervous system may play a key role in cardiovascular autonomic dysfunction due to oxidative stress.

Reactive oxygen species, especially superoxide derived from NADPH oxidase reacts with and inactivates $\mathrm{NO}$ and thereby modulates its bioavailability. NO in the brainstem, particularly in the Nucleus of Tractus Solitarius (NTS) and Rostral Ventrolateral Medulla (RVLM), exerts a tonic inhibitory influence on sympathetic outflow. Reduced NO in the RVLM enables an enhanced sympathetic discharge by decreased local GABA-ergic inhibitory inputs ${ }^{35,36}$. So higher oxidative stress could cause alteration in central sympathetic outflow in PCOS patients by the above mechanisms.

This study demonstrated exaggerated sympathetic response in the present series of PCOS patients when compared to control. As none of the measures of sympathetic reactivity were found abnormal in the PCOS patients there was no evidence of autonomic neuropathy in the patient group. In addition, increased oxidative stress, supported by lower plasma catalase and higher plasma MDA was found associated with PCOS patients in this study. Moreover, sympathetic autonomic reactivity in the current series of PCOS patients was associated with higher oxidative stress.

Conclusion

This study concluded that exaggerated sympathetic autonomic reactivity were related to higher oxidative stress in PCOS patients.

\section{Conflict of interest None}

\section{References}

1. Bozdag G, Mumusoglu S, Zengin D, Karabulut E, Okan Y. The prevalence and phenotypic features of polycystic ovary syndrome: a systematic review and meta-analysis. Hum Reprod 2016; 31(12): $2841-2855$.

Volume 16 No. 2 December 2021: 61-69
2. Franks S. Polycystic ovary syndrome: not just a fertility problem. Women's Health 2015; 11(4): 433-436.

3. Lansdown A, Rees DA. The sympathetic nervous system in polycystic ovary syndrome: a novel therapeutic target? Clin Endocrinol2012; 77: 791801 .

4. Rotterdam ESHRE/ASRM-Sponsored PCOS consensus workshop group. Revised 2003 consensus on diagnostic criteria and long-term health risks related to polycystic ovary syndrome (PCOS). Hum Reprod 2004; 19 (1): 41-47.

5. Saranya K, Pal GK, Habeebullah S, Pal P. Cardiovascular Risks and Sympathovagal Imbalance in Polycystic Ovarian Syndrome. Int J Biol Sci Opin 2012; 1(1): 192-201.

6. Thayer JF, Yamamoto SS, Brosschot JF. The relationship of autonomic imbalance, heart rate variability and cardiovascular disease risk factors. Int J Cardiol2010; 141(2):122-131.DOI:10.1016/ j.ijcard.2009.09.543.

7. Hashim ZH, Hamdan FB, Al-Salihi AR. Autonomic dysfunction in women with polycystic ovary syndrome. Iran J Reprod Med 2015; 13(1): 27-34.

8. Saranya K, Pal GK, Habeebullah S, Pal P . Assessment of cardiovascular autonomic function in patients with polycystic ovary syndrome. J Obstet Gynaecol Res 2014; 40 (1): 192-199. DOI: 10.1111/ jog. 12154

9. De Sa JC, Costa EC, Da Silva E, Zuttin RS, Da Silva EP, Lemos TM. Analysis of heart rate variability in polycystic ovary syndrome. Gynecol Endocrinol 2011; 27: 443-447.

10. Tekin G, Tekin A, Kilicarslan EB, Haydardedeoðlu B, Katircibapi A, Koçum T, Erol T, Çölkesen Y, Sezgin AT, Müderrisoðlu H . Altered autonomic neural control of the cardiovascular system in patients with polycystic ovary syndrome. Int $\mathrm{J}$ Cardiol 2008; 130 (1): 49-55.

11. Yildirir A, Aybar F, Kabakci G, Yarali H, Oto A. Heart rate variability in young women with polycystic ovary syndrome. Ann Noninvasive Electrocardiol 2006; 11(4): 306-312.

12. Saranya K, Pal GK, Habeebullah S, Pal P. Assessment of cardiovascular autonomic reactivity in patients with polycystic ovary syndrome. J Biol Sci Opin 2014; 2(3): 243-247. 
13. Zanella A, Sarolo L, Pomerri F, Bilora F. Autonomic dysfunction and flow-mediated dilation in polycystic ovary syndrome (PCOS): a case-control study. Dysautonomia in polycystic ovary syndrome. J Gynaecol Obstet 2016; 28(N1): 19-26.

14. Kilit C, Kilit TP. Heart rate variability in normalweight patients with polycystic ovary syndrome. Anatol J Cardiol 2017; 17(5): 404-409.

15. Özkeçeci G, Ünlü BS, Dursun H, Akçi O, Köken G, Onrat E, $A v^{0}$ ar A. Heart rate variability and heart rate turbulence in patients with polycystic ovary syndrome. Anatol J Cardiol 2016; 16(5): 323-327.

16. Ewing DJ, Clarke BF. Diagnosis and management of diabetic autonomic neuropathy. Br Med J 1982; 285(6346): 916-918.

17. Kandasamy S, Sivagamasundari RI, Bupathy A, Sethubathy S, Gobal V. Evaluation of insulin resistance and oxidative stress in obese patients with polycystic ovary syndrome. IJABPT 2010; 1(2): 391-398.

18. Fenkci V, Fenkci S, Yilmazer M, Serteser M. Decreased total antioxidant status and increased oxidative stress in women with polycystic ovary syndrome may contribute to the risk of cardiovascular disease. Fertil Steril 2003; 80 (1): 123-127.

19. Desai V, Prasad NR, Manohar SM, Sachan A, Narasimha SR, Bitla AR. Oxidative stress in nonobese women with polycystic ovarian syndrome. J Clin Diagn Res 2010; 8(7): CC01-3.DOI: 10.7860/ JCDR/2014/8125.4530

20. Kuscu NK, Var A. Oxidative stress but not endothelial dysfunction exists in non-obese, young group of patients with polycystic ovary syndrome. Acta Obstetricia Gynecologica Scand 2009; 88(5): 612-617.

21. Ighodharo OM, Akinloye OA. First line defense antioxidants-superoxide dismutase (SOD), catalase (CAT) and glutathione peroxidase $\left(\mathrm{GP}_{\mathrm{x}}\right)$ : Their fundamental role in the entire antioxidant defense grid. Alexandria J Med 2018; 54(4): 287-293.

22. Sabuncu T, Vural T, Harma M and Harma M. Oxidative stress in polycystic ovary syndrome and its contribution to the risk of cardiovascular disease. Clin Biochem2001; 34(5): 407-413.

23. Özer A, Bakacak M, Kiran H, Ercan Ö, Köstü B, Pektap MK, Kýlýnç M, Aslan F. Increased oxidative stress is associated with insulin resistance and infertility in polycystic ovary syndrome. Ginekol Pol 2016; 87(11): 733-738.

24. Rasool M, Ahmad R, Rizwan R, Malik A, Asif M, Zaheer A, Jabbar A, Zain M, Qaisrani MM, Qaisrani TB, Mehmood A, Malik IR, HelmiN, Zeyadi M, Choudhry H, Harlev A, Agarwal A, Jamal MS. Interrelationship of circulating biochemical markers of oxidative stress and comorbid condition in polycystic ovary syndrome. Biomed Res 2018; 29(21): 3779-3783.

25. Dos Santos ACS, Azevedo GD, Lemos TMAM. The Influence of Oxidative Stress in Inflammatory Process and Insulin Resistance in Obese Women with Polycystic Ovary Syndrome. Transl Biomed. 2016; 7(4): 1-4.

26. Mohan SK, Priya V. Lipid peroxidation, glutathione, ascorbic acid, vitamin E, antioxidant enzyme and serum homocysteine status in patients with polycystic ovary syndrome. Biol Med 2009; 1(3): 44-49.

27. Fadaee SB, Beetham KS, Howden EJ, Stanton T, Isbel NM, Coombes JS. Oxidative stress is associated with decreased heart rate variability in patients with chronic kidney disease. Redox Report 2017; 22(5): 197-204

28. Pavithran P, Nandeesha H, Sathiyapriya V, Bobby Z, Madanmohan T. Short-Term Heart Variability and Oxidative Stress in Newly Diagnosed Essential Hypertension. Clin Exp Hypertens 2008; 30(7): 486-496.

29. Western Pacific Region (WPRO), World Health Organization. The Asia Pacific Perspective: Redefining Obesity and its Treatment. [Internet] [Cited 26th march 2019]. Available from iris.wpro.who.int/handle/10655.1/5379

30. Gyftaki R, Gougoura S, Kalogeris N, Loi V, Koukoulis G, Vryonidou A. Oxidative stress markers in women with polycystic ovary syndrome without insulin resistance. Endocrine Abstracts 2018; 56 P950. DOI:10.1530/endoabs.56.P950

31. Bausenwein J, Serke H, Eberle K, Hirrlinger J, Jogschies P, Abu Hmeidan F, Blumenauer V, SpanelBorowski K. Elevated levels of oxidized low-density lipoprotein and of catalase activity in follicular ûuid of obese women. Mol Hum Reprod 2010; 16(2):117-124.DOI: 10.1093/molehr/gap078

32. Erdogan M. Karadeniz M. Berdeli A. Alper G. Caglayan O, Yilmaz C. The relationship of the

Volume 16 No. 2 December 2021: 61-69 
interleukin-6 -174 G>C gene polymorphism with oxidative stress markers in Turkish polycystic ovary syndrome patients. J Endocrinol Invest 2008; 31: 624-629.

33. Karadeniz M, Erdoðan M, Tamsel S, Zengi A, Alper GE, Caðlayan O, Saygili F, Yilmaz C. Oxidative stress markers in young patients with polycystic ovary syndrome, the relationship between insulin resistances. Exp Clin Endocrinol Diabetes 2008; 116 (4): 231-235. DOI: 10.1055/s-2007-992154.

34. Dursun P, Demirta ${ }^{\circ}$ E, Bayrak A, Yarali H. Decreased serum paraoxonase 1 (PON1) activity: an additional risk factor for atherosclerotic heart disease in patients with PCOS? Hum Reprod 2006; 21(1): 104-108.

35. Hirooka Y, Kishi T, Sakai K, Takeshita A, Sunagawa $\mathrm{K}$. Imbalance of central nitric oxide and reactive oxygen species in the regulation of sympathetic activity and neural mechanisms of hypertension. Am J Physiol Regul Integr Comp Physiol 2011; 300: R818-R826 DOI:10.1152/ajpregu. 00426. 2010

36. Zanzinger J.Role of nitric oxide in the neural control of cardiovascular function. Cardiovasc Res 1999; 43(3): 639-649. 